\title{
Acute polyradiculoneuritis with locked-in syndrome in a patient with Covid-19
}

\author{
Thomas Pfefferkorn ${ }^{1}$ (1) - Rainer Dabitz ${ }^{1} \cdot$ Thomas von Wernitz-Keibel $^{2} \cdot$ Johannes Aufenanger $^{3}$. \\ Martina Nowak-Machen ${ }^{2} \cdot$ Hendrik Janssen ${ }^{4}$
}

Received: 21 April 2020 / Revised: 3 May 2020 / Accepted: 5 May 2020 / Published online: 12 May 2020

○) Springer-Verlag GmbH Germany, part of Springer Nature 2020

\section{Background}

Acute neuropathy in patients with Covid-19 is increasingly reported [1-6]. We present another case with fulminant polyradiculoneuritis, suggestive of Guillain-Barré-syndrome (GBS), leading to a locked-in syndrome with corresponding generalized nerve root contrast enhancement on MRI.

\section{Case}

A 51-year-old male patient was referred to our hospital on April 2nd, 2020 with a 2 day history of progressive upper and lower limb weakness and acral paresthesias. He also reported fluctuating fever and flu-like symptoms with marked fatigue and dry cough over the last 2 weeks.

On clinical examination, substantial tetraparesis with limb muscle strength ranging from $2 / 5$ to $4 / 5$ (MRC grading scale) as well as generalized areflexia were documented. Vital signs were stable at that point.

A low dose chest CT scan upon admission revealed typical bilateral interstitial infiltrates in keeping with Covid-19 pneumonia. Covid-19 infection was then confirmed by a SARS-CoV-2 positive PCR testing result from a pharyngeal swab.

Thomas Pfefferkorn

thomas.pfefferkorn@klinikum-ingolstadt.de

1 Department of Neurology, Klinikum Ingolstadt, Krumenauerstrasse 25, 85049 Ingolstadt, Germany

2 Department of Anaesthesiology and Intensive Care Medicine, Klinikum Ingolstadt, Ingolstadt, Germany

3 Institute of Laboratory Medicine, Klinikum Ingolstadt, Ingolstadt, Germany

4 Institute of Neuroradiology, Klinikum Ingolstadt, Ingolstadt, Germany
Cerebrospinal fluid (CSF) on admission revealed mild pleocytosis ( 9 cells/ $\mu \mathrm{l})$, normal protein content and negative PCR testing for SARS-CoV-2. Serum anti-ganglioside antibodies were negative.

Electroneurography on the day after admission demonstrated prolonged distal motor latencies (left median nerve $8.4 \mathrm{~ms}$; left tibial nerve $11.6 \mathrm{~ms}$ ) and loss of F waves suggesting peripheral demyelination.

Due to a rapidly deteriorating pulmonary gas exchange the patient needed endotracheal intubation on day two after admission. After pulmonary stabilization tracheostomy was performed on day seven after admission and weaning from the ventilator attempted.

Despite treatment with intravenous immunoglobulins (IVIG, $30 \mathrm{~g}$ daily for 5 days starting upon admission) the neurological status deteriorated to an almost complete peripheral locked-in syndrome with tetraplegia, complete sensory loss in all extremities, bilateral facial and hypoglossal paresis as well as ongoing respiratory failure due to muscular weakness.

In light of the fulminant neurological course plasma exchange therapy (PE) was begun on day 13 after admission (total of 14 treatments). Just prior to initiation of $\mathrm{PE}$ a second CSF sample was taken which now demonstrated marked protein elevation $(10.231 \mathrm{mg} / \mathrm{l}$; normal range up to $450 \mathrm{mg} / \mathrm{l})$ in the absence of pleocytosis.

An MRI of the spine was performed on day 14 after admission and demonstrated massive symmetrical contrast enhancement of the spinal nerve roots at all levels of the spine including the cauda equina. Interestingly anterior and posterior nerve roots were equally affected (Fig. 1).

Thirty-one days after admission the patient was referred to a specialized rehabilitation clinic. At that time he showed signs of motor improvement with regressive facial and hypoglossal paresis but still needed mechanical ventilation. 

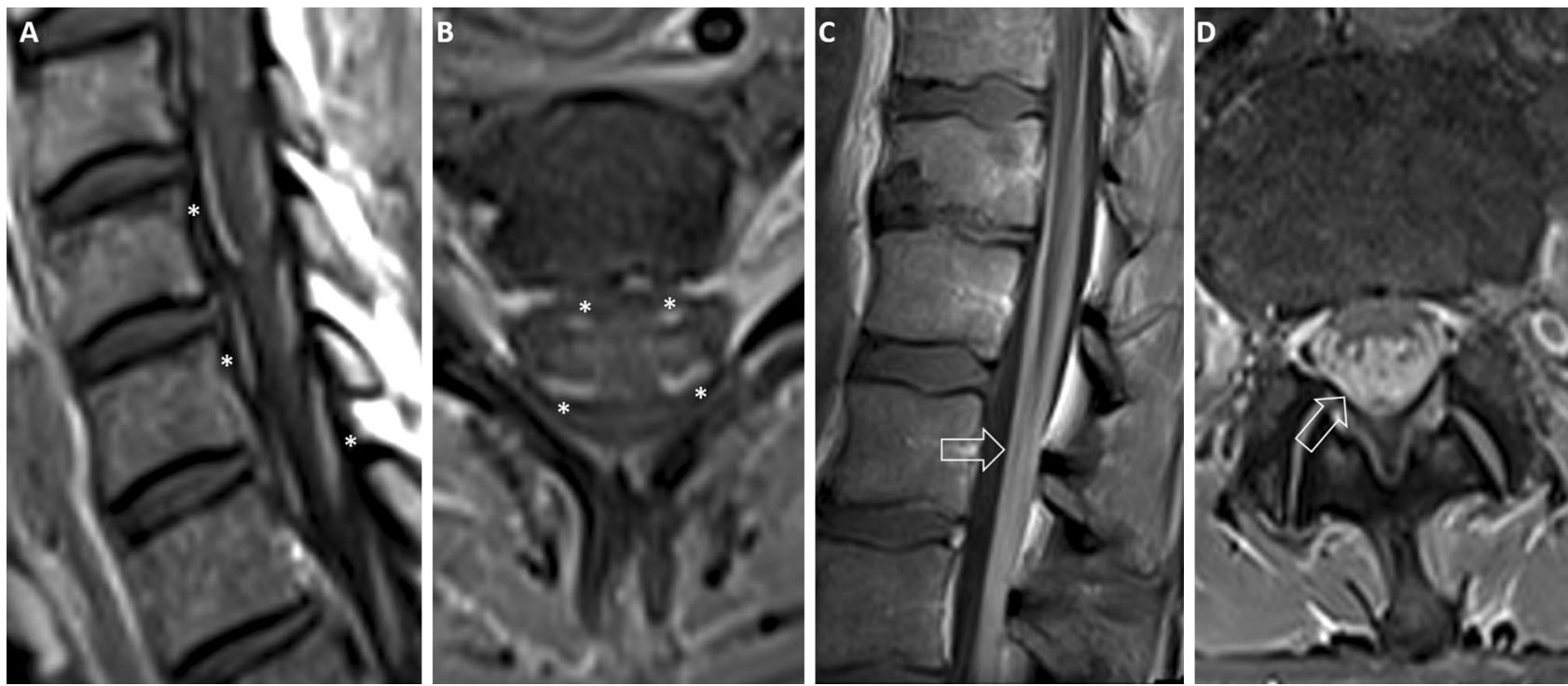

Fig. 1 MRI (T1 weighted sequences after application of gadolinium) shows symmetrical enhancement of the anterior and posterior roots (white *) in the cervical spine (a, b) and the cauda equina (c,d) (white arrow)

\section{Discussion}

In the presented Covid-19 patient, the clinical diagnosis of acute polyradiculoneuritis, highly suggestive of para-infectious GBS, was confirmed by spinal MRI.

The complete sensory loss in our patient, which is considered uncommon in GBS, corresponds to the extensive posterior nerve root involvement, the ongoing neurogenic respiratory failure to the cervical root involvement. Since respiratory failure is known to be a common and hazardous problem in acute Covid-19 patients, high cervical polyradiculoneuritis should be considered a possible cause in patients developing limb weakness or cranial nerve involvement.

IVIG and PE are the two treatment options in moderate to severe GBS. We started with IVIG therapy right upon admission, expecting a low risk of harm with regards to Covid19. With exacerbating neurological symptoms, a therapeutic switch to PE was considered early but initially not performed due to concern to remove the just administered IVIG and to compromise the patient's immunocompetence in active Covid-19 pneumonia. PE was started only on day 13 after admission, when pulmonary gas exchange had normalized and a SARS-CoV-2 tracheal specimen was tested negative.

Our case provides further evidence that GBS is a relevant complication of Covid-19. The value of IVIG and PE needs to be further evaluated in this specific setting.

\section{Compliance with ethical standards}

Conflicts of interest There are no conflicts of interest.
Ethical standards For this type of study formal consent is not required.

\section{References}

1. Camdessanche J-P, Morel J, Pozzetto B, Paul S, Tholance Y, Botelho-Nevers E (2020) COVID-19 may induce Guillain-Barré syndrome. Revue Neurologique. https://doi.org/10.1016/j.neuro 1.2020 .04 .003

2. Gutiérrez-Ortiz C, Méndez A, Rodrigo-Rey S, Pedro-Murillo ES, Bermejo-Guerrero L, Gordo-Mañas R et al (2020) Miller Fisher Syndrome and polyneuritis cranialis in COVID-19. Neurology. https://doi.org/10.1212/WNL.0000000000009619

3. Padroni M, Mastrangelo V, Asioli GM, Pavolucci L, AbuRumeileh S, Piscaglia MG et al (2020) Guillain-Barré syndrome following COVID-19. New infection, old complication? J Neurol 395(10229):1054. https://doi.org/10.1007/s00415-020-09849-6

4. Sedaghat Z, Karimi N (2020) Guillain Barre syndrome associated with COVID-19 infection. A case report. J Clin Neurosci. https:// doi.org/10.1016/j.jocn.2020.04.062

5. Toscano G, Palmerini F, Ravaglia S, Ruiz L, Invernizzi P, Cuzzoni MG et al (2020) Guillain-Barré Syndrome associated with SARSCoV-2. N Engl J Med. https://doi.org/10.1056/NEJMc2009191

6. Virani A, Rabold E, Hanson T, Haag A, Elrufay R, Cheema T et al (2020) Guillain-Barré Syndrome associated with SARS-CoV-2 infection. IDCases 20:e00771. https://doi.org/10.1016/j.idcr.2020. e00771 